\title{
Biologics, Pharmacovigilance, and Patient Safety: It's All in the Name
}

\author{
James G. Stevenson, PharmD, FASHP, and Larry Green, PharmD, FASHP
}

\begin{abstract}
SUMMARY
The most appropriate naming convention for biologics and biosimilars has been an area of significant debate. The ultimate decision will have an impact on patient safety, pharmacovigilance program effectiveness, and, potentially, the overall adoption of biosimilars in the United States. This article reviews some of the advantages and disadvantages of various naming approaches. For clarity in communication, optimal pharmacovigilance, and patient safety, it is recommended that biosimilars be named with a common USAN (United States Adopted Name) with the reference product, along with a suffix that is memorable, such as one associated with the original manufacturer of the product. This approach supports the FDA's mission of protecting patient safety and public health, while minimizing the possibility of inadvertent switching of products and facilitating effective pharmacovigilance.
\end{abstract}

J Manag Care Spec Pharm. 2016;22(8):927-30

Copyright $\odot 2016$, Academy of Managed Care Pharmacy. All rights reserved.

$\mathrm{I}$ $\mathrm{n}$ this month's issue of the Journal of Managed Care $\mathcal{E}$ Specialty Pharmacy, Dr. Daniel Tomaszewski, of Chapman -University School of Pharmacy, reports on a survey of members of the Academy of Managed Care Pharmacy (AMCP) and the Hematology Oncology Pharmacists Association, which was conducted in May-June 2015, before the U.S. Food and Drug Administration (FDA) released its proposed guidance on biosimilar naming. ${ }^{1}$ Although the survey response rate was low, those who did respond expressed a preference for a distinct name for biosimilars, rather than having them share the same nonproprietary name as the reference biologic. Specifically, the survey respondents preferred the use of a nonproprietary name with a designated suffix over other potential distinct naming options, such as a nonproprietary name plus a prefix or reliance on a unique brand name. However, of those respondents who preferred a nonproprietary name plus a suffix, the majority (83.4\%) preferred a suffix tied to the manufacturer, compared with $16.6 \%$ of the respondents who preferred a suffix devoid of meaning.

While, presumably, the preference for a distinct name for biosimilars has been motivated by the need for strong pharmacovigilance programs, this survey also found that pharmacists reported being more confident in substituting interchangeable biosimilars when they share a nonproprietary name and that confidence in substitution decreases when a unique name, such as a nonproprietary name plus a suffix, is used in naming an interchangeable biosimilar. However, the differences were not large. The results of this survey are similar to those of an earlier survey by Fernandez-Lopez et al. (2015), which canvassed pharmacists who were members of AMCP, the American Society of Health-System Pharmacists, and the American Pharmacists Association. ${ }^{2}$ Also in 2015, the Alliance for Safe Biologic Medicines found that $68 \%$ of pharmacists supported distinct nonproprietary scientific names, and $77 \%$ supported memorable suffixes based on manufacturer names. ${ }^{3}$

\section{Biosimilar Approval and FDA Draft Guidance}

The first biosimilar in the United States was approved with the name filgrastim-sndz (Zarxio), with a common United States Adopted Name (USAN) and a suffix (sndz) that reflected the manufacturer (Sandoz). The second biosimilar approved by the FDA was infliximab-dyyb (Inflectra) manufactured by Celltrion. In this case, the name was a common USAN with an apparently random suffix devoid of meaning. In August 2015 (between the 2 biosimilar approvals), the FDA released its draft guidance on the naming of biological products. ${ }^{4}$ This draft guidance calls for all biologics to be named with a "core name," which the FDA intends to be the USAN, if there is one, plus a designated hyphenated suffix that is unique and devoid of meaning or reference to the manufacturer. This is a similar approach to that recommended by the World Health Organization (WHO). ${ }^{5} \mathrm{~A}$ rationale provided by the FDA in its draft guidance document for this naming convention is the need for a method of distinguishing between biological products that are not interchangeable for pharmacovigilance purposes. This rationale is consistent with views expressed by pharmacists whose priority is the protection of patient safety and public health.

\section{Use of Suffixes}

Adoption of the naming convention in the FDA draft guidance would involve renaming already approved reference biological products by adding a suffix. Some have argued that requiring suffixes for only biosimilars and not reference products could lead to a perception of inferior safety and efficacy for biosimilars or that they differ in clinically meaningful ways, which could hinder their acceptance by providers and patients. This point seems to be confirmed by the Tomaszewski study, which found that surveyed pharmacists would have the greatest confidence in substituting interchangeable biosimilars if they shared the same nonproprietary name as the reference product. ${ }^{1}$ Since biosimilars could be approved for fewer indications than the reference product and could have fewer approved routes of administration, the FDA felt that sharing the same nonproprietary name could lead to inadvertent substitution and medication errors and that designation of a unique proper 
name (USAN plus suffix) would be the best mechanism to facilitate safe use. The use of a suffix (instead of a prefix) is preferred by the FDA because it would allow products with the same core name to be grouped together in electronic databases and systems for ordering, dispensing, and administering medications. In its draft guidance, the FDA stated that it is still considering whether interchangeable products should share the same suffix as the reference product. Certainly, any naming convention should facilitate pharmacovigilance and prevent inadvertent switching of products as a means of ensuring the success of the biosimilar pathway and not deterring the uptake of products.

\section{Areas of Concern}

On June 2, 2016, the Office of Management and Budget (OMB) published a request for comment on the workload associated with the FDA's "Nonproprietary Naming of Biological Products: Guidance for Industry." ${ }^{4,6}$ This notice indicates that the FDA has submitted a naming guidance for publication. This is a prerequisite to publishing the naming guidance, but at this point, there is no indication when the guidance will be published or whether the guidance will be final or another draft requested.

While many organizations support the idea of a distinct name for biosimilars for pharmacovigilance purposes, ${ }^{7} 2$ key areas of concern have been articulated. First, there is still a lack of consensus as to whether interchangeable biosimilars should share the same core name and suffix as the reference product. To some extent, the preference of surveyed pharmacists for identical proper names for interchangeable biologics may stem from familiarity with state pharmacy practice acts applying to multisource generics. State laws often restrict substitution to generic products using the same generic name or containing the same active ingredient. While sharing a common core name and suffix could be a tool to identify a biosimilar as being interchangeable - and some have argued that it might facilitate the adoption and uptake of approved biosimilars-there is still the problem of differentiating interchangeable biological products for pharmacovigilance purposes. In addition, market research in international markets indicate that distinguishable nonproprietary names for biosimilars do not negatively impact market acceptance. ${ }^{8}$ Second, there is concern regarding the FDA's recommendation to use a suffix for biologics and biosimilars that is "devoid of meaning."

\section{Specificity or Sharing?}

Even with biosimilars that may eventually be designated as interchangeable by the FDA, there is a need for specificity in naming to facilitate identification because an adverse event may be specific to the product but not to the entire class of products. Grampp and Felix (2015) clearly outlined the rationale for the use of distinctive names for biosimilars for pharmacovigilance purposes. ${ }^{9}$ In order for spontaneous reporting systems for adverse events and active surveillance systems to be effective, there must be a reliable mechanism to correctly identify a product that has been dispensed or administered to a patient. This is particularly true for the pharmacovigilance of biologics, since there is the potential for delayed immunologic reactions as a result of the development of antidrug antibodies. The time period between the administration of a specific product and the development of an adverse event, or change in efficacy, may complicate identification of the specific product involved, particularly in a patient who has received multiple versions of a biologic.

The importance of distinctive product identification was demonstrated in a study of the FDA Adverse Event Report System database. ${ }^{10}$ This study showed that for 6 of 8 small molecule drugs evaluated, the number of adverse events attributed to the reference product did not decrease significantly during a time when generic availability occurred, and there was a corresponding significant decrease in the volume of prescriptions for the brand reference product. ${ }^{10}$ This finding suggests that the adverse events were incorrectly attributed to the brand product that shared the generic drug's nonproprietary name, even though a generic product was actually administered.

If biosimilars were to share a common nonproprietary name with the reference biologics, other options could be considered to differentiate the products for pharmacovigilance purposes. One option is the use of different brand names, which is the process used in the European Union. However, this process seems to fall short of full traceability. Despite legislation that requires member states to ensure that brand names are used in health records, $21 \%$ of European-sourced spontaneous reports for filgrastim adverse events submitted to the originator product sponsor were not attributed to a specific brand name. ${ }^{11}$

The National Drug Code (NDC) has also been suggested as a potential method of differentiating biological products. While NDC numbers are commonly included in outpatient prescription drug claims and could be used to differentiate products dispensed through this channel, they are not often used in physician office or infusion center billing systems. Rather, these systems often use Healthcare Common Procedure Coding System (HCPCS) codes to identify drugs that are administered in these settings. The majority of biosimilars currently in development are administered in a physician's office or infusion center as opposed to the outpatient channel. Also, while an enhanced NDC number contains the necessary information needed for accurate pharmacovigilance activities, current proposals to include NDC numbers in information systems used by all providers in all health care settings is years away. To further complicate matters from a pharmacovigilance perspective, the Centers for Medicare \& Medicaid Services have proposed that all biosimilars share a common J-code, the HCPCS code generally used for infusions and injections. ${ }^{12}$ 
The introduction of generic enoxaparin illustrates the potential problems with accurate identification of the correct drug product. After generic enoxaparin had captured $50 \%$ of the market, adverse drug events attributable to specific generic enoxaparin products were roughly 9-fold lower than expected. While claims from a pharmacy benefit identified with a specific NDC number were less problematic, claims under a medical benefit were very unreliable in identifying the correct product. ${ }^{13}$

This experience supports the need articulated in the FDA naming guidance for distinct nonproprietary names for biosimilars and reference biological products. The enoxaparin experience also suggests that even interchangeable biosimilars should have distinct names, since accurate pharmacovigilance is unlikely to occur without a naming convention that can distinguish between interchangeable products, unless some profound change is made to coding and billing requirements (e.g., the requirement of an 11-digit NDC number on all pharmacy and medical claims and in hospital dispensing records). Any such change would take many years for adoption and implementation. For interchangeable biosimilars, prescribers and pharmacists will need to rely on the FDA's Purple Book to distinguish between biosimilars and interchangeable biosimilars when considering substitution policies and regulations. ${ }^{14}$

\section{Recommendations}

Assigning biosimilars and interchangeable biosimilars a nonproprietary name with a meaningful suffix would be the best approach to minimize inadvertent substitution as well as to facilitate pharmacovigilance. There is significant concern regarding the proposed guidance from the FDA and WHO that calls for a suffix "devoid of meaning." The FDA's rationale for this approach is that a suffix indicating the licensee would be problematic if the product were to be sold to another manufacturer or in the case of corporate mergers or acquisitions. We believe that the use of suffixes "devoid of meaning" would lead to significant unintended consequences, some of which may actually harm overall pharmacovigilance efforts. While the use of a suffix that indicates the manufacturer of the product would be understood by providers and patients, the use of a suffix devoid of meaning would likely cause confusion and difficulty in the identification and communication of the precise product that a patient would be receiving. The use of this type of suffix would be particularly problematic when attempting to accurately identify products in spontaneous adverse event reporting systems. It would also likely be a significant problem in transitions of care when patients move from one place of care to another. Confusion or ambiguity in the communication of the specific product that a patient is receiving will likely result in inadvertent switching of products and delays in the conduct of critical safety research, particularly with the diverse nature and current limitations in health information systems. Furthermore, we are concerned that the use of a common USAN plus a unique designated suffix devoid of meaning would mean that similar biological products will be grouped together by the common name in electronic prescribing, dispensing, and administration systems. Distinguishing the correct product by viewing a list of several products with a common USAN but with suffixes that have no logical meaning will be potentially confusing and will likely result in product selection errors. It would be more likely that the correct product would be selected and also communicated accurately if the suffix was memorable and had meaning, for example, reflecting the original manufacturer of the specific product. Although there might be issues if the name of the manufacturer of a product changed or if the manufacturer was acquired by another entity, a product name that includes a suffix with some logical meaning would still be much less confusing for patients and providers to deal with than with the proposed naming guidance for a suffix devoid of meaning. This point is clearly illustrated in the Tomaszewski survey, which reported that over $83 \%$ of pharmacists who preferred a distinct name for biosimilars also preferred a USAN plus a suffix tied to a manufacturer.

\section{Conclusions}

Prescribers and pharmacists will play an important role in the safe and appropriate use of biosimilars, as well as promoting their uptake. We urge all stakeholders to clearly consider this important issue of biosimilar naming from a pharmacovigilance and patient safety perspective to ensure protection of public health. We believe that the best option for clarity in communication, optimal pharmacovigilance, and patient safety is to require that biosimilars be named with a common USAN with the reference product, along with a suffix that is memorable, such as association with the manufacturer of the product. This approach supports the FDA's mission of protecting patient safety and public health, minimizing inadvertent switching of products, and facilitating pharmacovigilance. In the event that the proposed naming convention includes a suffix devoid of meaning is adopted, it is recommended that products be listed in electronic systems with the USAN/suffix plus a brand name or manufacturer. This will increase clarity for health care providers and should result in fewer wrong product selection errors. Furthermore, patients should be counseled on the brand name and manufacturer of the product that they are taking in order to improve accuracy in medication reconciliation and to avoid inadvertent switching.

\section{Authors}

JAMES G. STEVENSON, PharmD, FASHP, University of Michigan College of Pharmacy, Ann Arbor, Michigan, and LARRY GREEN, PharmD, FASHP, Amgen, Inc., Thousand Oaks, California.

AUTHOR CORRESPONDENCE: James G. Stevenson, PharmD, FASHP, University of Michigan College of Pharmacy, 428 Church St., Ann Arbor, MI 48109. Tel.: 248.894.3435.E-mail: jimsteve@umich.edu. 


\section{DISCLOSURES}

No funding supported the writing of this article. Stevenson reports consulting fees from Amgen, Inc., AbbVie, and Pfizer and is employed by Visante. Green is employed by and owns stock in Amgen, Inc.

Article concept was contributed primarily by Stevenson, along with Green. The manuscript was written and revised primarily by Stevenson, along with Green. Both authors contributed equally to data interpretation.

\section{REFERENCES}

1. Tomaszewski D. Biosimilar naming conventions: pharmacist perceptions and impact on confidence in dispending biologics. J Manag Care Spec Pharm. 2016;22(8):919-26.

2. Fernandez-Lopez S, Kazzaz D, Bashir M, McLaughlin T. Assessment of pharmacists' views on biosimilar naming conventions. J Manag Care Spec Pharm. 2015;21(3):188-95. Available at: http://www.jmcp.org/doi/10.18553/ jmcp.2015.21.3.188.

3. Olson K. Biosimilars naming and labeling: a study of U.S. pharmacists. Alliance for Safe Biologic Medicines. October 2015. Available at: https://safebiologics.org/wp-content/uploads/2016/04/2015-US-Pharmacists-Survey-1. pdf. Accessed July 7, 2016.

4. U.S. Food and Drug Administration. Nonproprietary naming of biological products. Guidance for industry (Draft guidance). Available at: http://www. fda.gov/downloads/drugs/guidancecomplianceregulatoryinformation/guidances/ucm459987.pdf. August 2015. Accessed July 7, 2016.

5. World Health Organization. Biological qualifier: an INN proposal. Programme on International Nonproprietary Names. INN Working Doc. 14.342. Revised June 2015. Available at: http://www.who.int/medicines/services/inn/bq_innproposal201506.pdf.pdf. Accessed July 7, 2016.

6. U.S. Food and Drug Administration. Notice submitted to Office of Management and Budget re proposed collection of information. 81 Fed Reg 35367. June 2, 2016. Available at: http://federalregister.gov/a/2016-12885. Accessed June 7, 2016.

7. Silverman E. Physician groups side with big drug makers over biosimilar names. The Wall Street Journal. August 15, 2014. Available at: http://blogs.wsj. com/pharmalot/2014/08/15/physician-groups-side-with-big-drug-makersover-biosimilar-names/. Accessed July 7, 2016.
8. Amgen Inc. Comment on the Food and Drug Administration (FDA) notice: nonproprietary naming of biological products; draft guidance for industry; availability. Posted October 27, 2015. Available at: https://www regulations.gov/\#!documentDetail;D=FDA-2013-D-1543-0166. Accessed July 7, 2016.

9. Grampp G, Felix T. Pharmacovigilance considerations for biosimilars in the USA. BioDrugs. 2015;29(5):309-21.

10. Lietzan EF, Sim LE, Alexander EA. Biosimilar naming: how do adverse event reporting data support the need for distinct non-proprietary names for biosimilars? Food Drug Policy Forum. 2013;3(6):1-24. Available at: http://www.fdli.org/docs/members-only/lietzan-faers-bio-final-3-27-13. pdf?sfvrsn=0. Accessed July 7, 2016.

11. Amgen Inc. Docket nos. FDA-2013-P-1153; FDA-2013-P-1398, respectively, non-proprietary naming of biosimilars. 2015. Available at: http:// www.regulations.gov/\#!documentDetail;D=FDA-2013-P-1398-0022. Accessed July 10, 2016

12. Centers for Medicare and Medicaid Services. Proposed rule re Medicare program: hospital outpatient prospective payment and ambulatory surgical center payment systems and quality reporting programs; short inpatient hospital stays; transition for certain Medicare-dependent, small rural hospitals under the hospital inpatient prospective payment system. 80 Fed Reg 39199. July 8, 2015. Available at: https://www.federalregister.gov/ articles/2015/07/08/2015-16577/medicare-program-hospital-outpatientprospective-payment-and-ambulatory-surgical-center-payment. Accessed July 7, 2016.

13. Grampp G. Bonafede M, Felix T, Li E, Malecki M, Sprafka JM. Active and passive surveillance of enoxaparin generics; a case study relevant to biosimilars. Expert Opin Drug Saf. 2015;14(3):349-60.

14. U.S. Food and Drug Administration. Purple Book: lists of licensed biological products with reference product exclusivity and biosimilarity or interchangeability evaluations. Available at: http://www.fda.gov/Drugs/ DevelopmentApprovalProcess/HowDrugsareDevelopedandApproved/ ApprovalApplications/TherapeuticBiologicApplications/Biosimilars/ ucm411418.htm. Accessed July 7, 2016. 